\title{
Smart Container- An Automated Alerting System
}

\author{
Gaurav Kodwani \\ Dept. of IT \\ CSPIT, CHARUSAT \\ Changa, India
}

\author{
Mayur Mehta \\ Dept. of IT, \\ CSPIT, CHARUSAT \\ Changa, India
}

\author{
Ayesha S. Shaikh \\ Asst. Prof., Dept. of IT, \\ CSPIT, CHARUSAT \\ Changa, India
}

\begin{abstract}
Many times there arises a situation during which containers filled with products are on a verge of completion and individuals are unaware of it. The containers which are filled with the bulk of products even gets completed and there arises an emergence of ordering that particular product in an abrupt manner. Containers at malls and at other storehouses sometimes get completed and due to unawareness over decrement in quantity lead to failure in production or transportation of that product in assigned time slot.

Hence in order to circumvent the above situation, A microcontroller based system is proposed in our paper that leads to aware an individual and give him/her track related to the decrement of product quantity filled in their respective containers or storage appliances. This IoT-based system comprises of container that is interfaced with IR sensor along with Arduino and GSM modem alerting over the actual completion of product quantity to respective entity. This smart system is capable of reducing human efforts of regular scrutinization over product quantity in the container along with enhancement of a smart city vision.
\end{abstract}

\section{Keywords}

Arduino, IR Sensor, GSM Modem, IoT, Line of Sight.

\section{INTRODUCTION}

The most tedious task for any shopping mall is to keep a track over the product's quantity which should be available for the customer all the time. For this purpose, the management department has to keep hiring employees in order to provide such services to the customers. This results in the increment of additional expenses over management purposes for the shopping mall.

From the customer's point of view, they get disappointed as they came from a long way home to buy some products and cannot find it in the mall due to employee's unawareness about the low quantity or unavailability of product in the container, hence this system is a competence towards their complication. This system can also be the part of the wellknown concept of 100 smart cities as initiated by our present Prime Minister of India, Sri Narendra Modi.

Talking just about alerting and automation is not enough, analysis of the product is also required when it comes to the business level. A system which is alerting the respective person via SMS about quantity can also do analysis, after collecting a good amount of data about how many times container was refilled in certain time intervals. Currently, this analysis is carried out by checking storage in the warehouse and keeping manual records of sells in stores or big shopping malls.

Our proposed system which is based on IoT (Internet of Things) provides following advantages to the user:
1. Alert on a predefined quantity of the product via SMS.

2. No need to keep track over the product quantity.

\section{LITERATURE SURVEY}

This idea is not indigenous; it has been implemented from the proposed system of smart dustbin which was used as an alerting system for garbage. Likewise, this system simply inherits the same properties of notifying about the quantity using the same methodology.

[1] IoT Based Smart Garbage and Waste Collection Bin as proposed by S.S.Navghane, M.S.Killedar, Dr.V.M.Rohokale insinuated us the idea of using IR sensor as a level detector for our container.

[2] Smart Dustbin-An Efficient Garbage Monitoring System as proposed by Monika K A, Nikitha Rao, Prapulla S B, Shobha G intimated us, how GSM modem is used to communicate with devices like cell phones that are forwarding us the details related to the quantity of product in our smart system.

[3] Smart Garbage Management System by Vikrant Bhor, Pankaj Morajkar, Maheshwar Gurav, Dishant Pandya. Here, the authors have given the detailed description of using intermediate hardware i.e. Arduino UNO between IR sensor and GSM modem and also, explained the functionality of GSM modem and IR sensor relative to their different components like pins, switch, power requirements, etc.

[4]Analysis of MAC Layer Issue using Application Layer Protocol for Smart Waste Management System by Kiran Patil and Dr. S. L. Lahudkar. By using multiple IR sensor and Raspberry $\mathrm{Pi}$, they have displayed the quantity in different figures. Also, they have used web services for transferring the data.

[5]SmartGarbage Monitoring System by Jetendra Joshi,Akshay, Amrit Bagga, Rahul Aggarwal, Manash Deka, Urjit, and Abhinav. They have incorporated many alerting systems in dustbins and have built a mobile application in order to keep track over garbage of multiple bins using web services. Also, if the limit of predefining quantity of garbage is crossed, the system will send respective notification into the application.

\section{PROPOSED SYSTEM}

\subsection{System Description}

Instead of performing manual exertion of keeping track over product quantity, our smart system automatically detects with the help of a sensor and alert via SMS. 


\subsection{Block Diagram of the System}

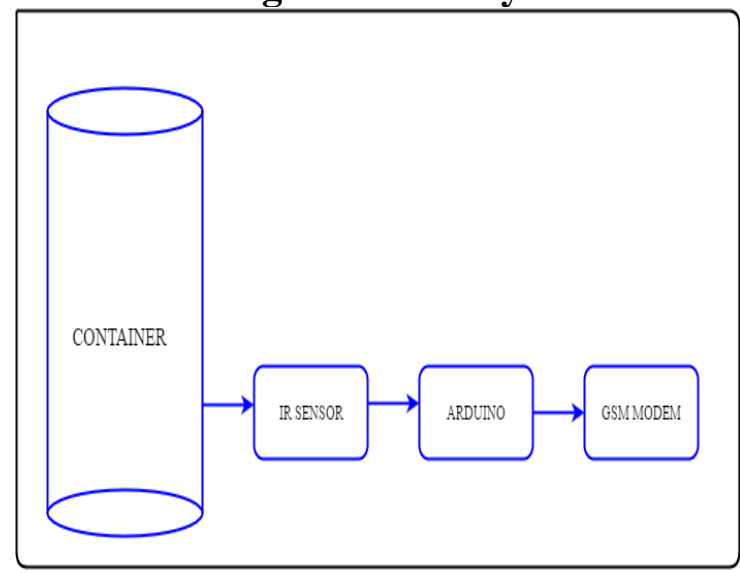

Fig 1: Flow diagram

\subsection{Components}

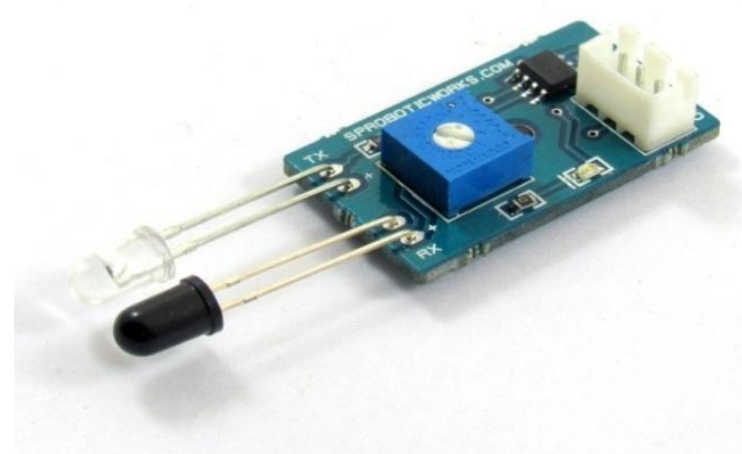

Fig 2: IR Sensor

IR Sensor: Figure 2 represent IR sensor which is an infrared sensor is an electronic device, having one emitter called IR LED (Light Emitting Diode) and the another one is detector called IR photodiode which is sensitive to IR light [4]. IR sensor is used to check whether the product is below the level of minimum requirement or not.

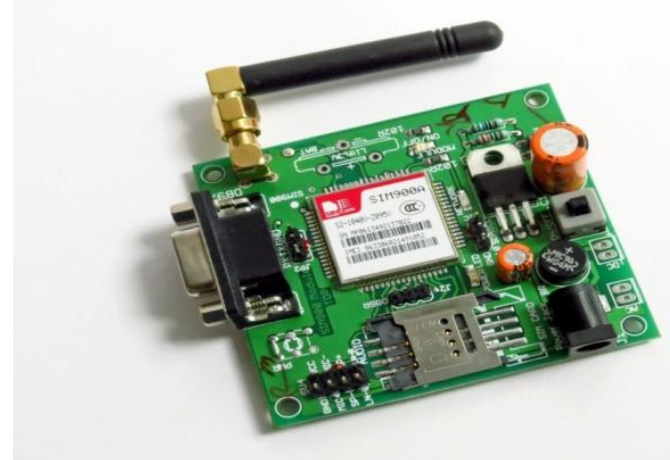

Fig 3: GSM Modem

GSM MODEM: Figure 3 represent GSM Modem which is a part of wireless MODEM devices which are used for communication over the wireless network [2]. It works with the help of SIM (Subscriber Identity Module) card which is registered with a mobile operator, similar to mobile phone.
Also, they have IMEI (International Mobile Equipment Identity) number just like every mobile device have. An external GSM modem is connected to Arduino through connecting wires. GSM Modem can make, receive or reject voice call, read, add, search phonebook entries of the SIM and receive, send or delete SMS messages in a SIM. GSM Modem transfer data using radio waves.

\section{Experimental Set and Outcome}

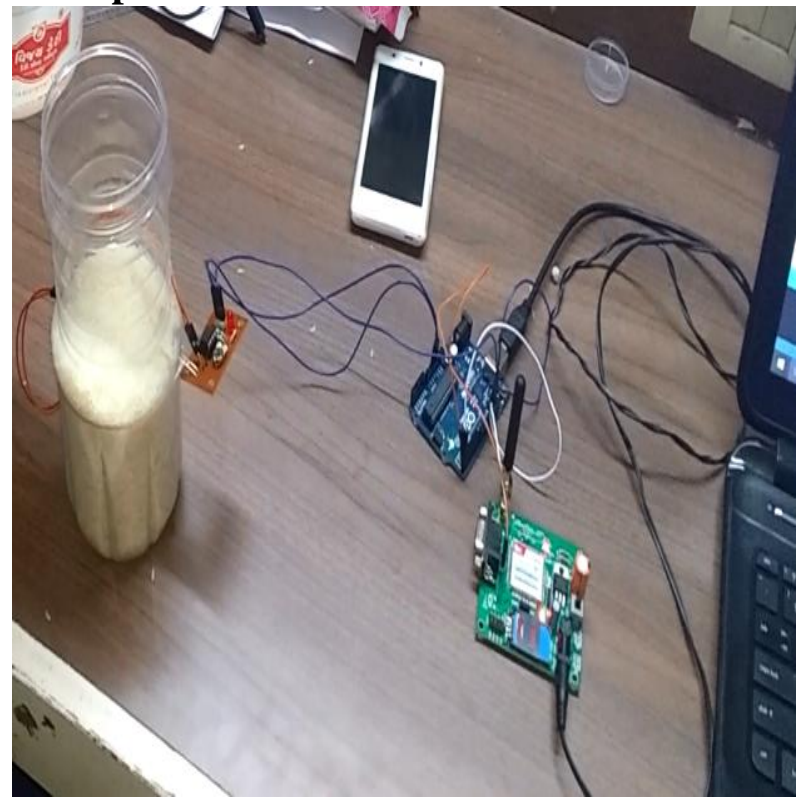

Fig 4: Hardware circuit

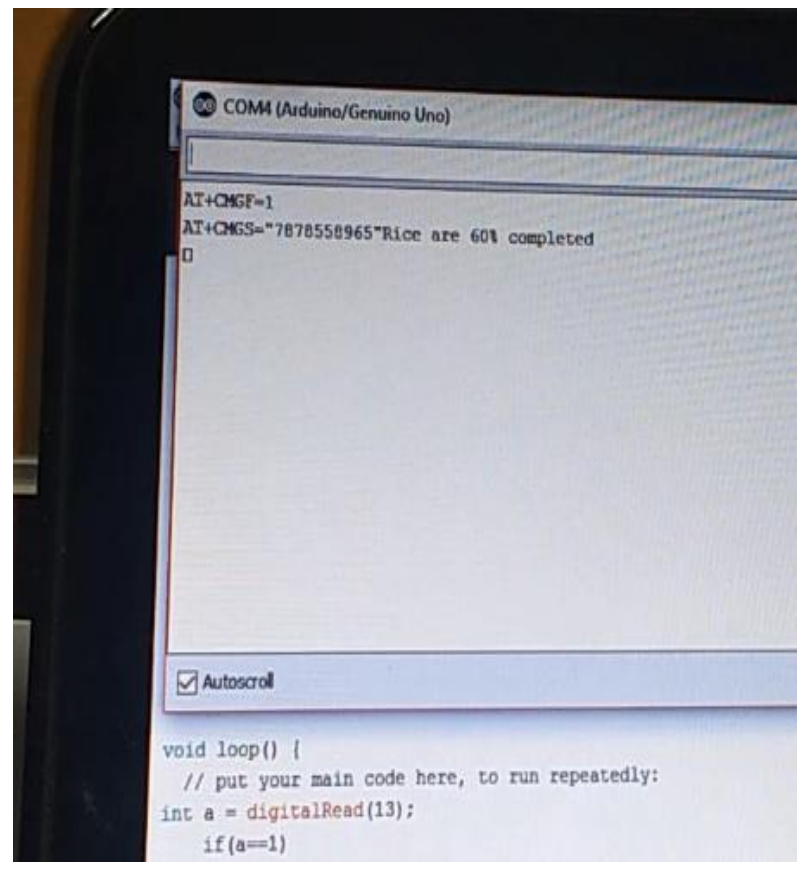

Fig 5: Output on COM Port 


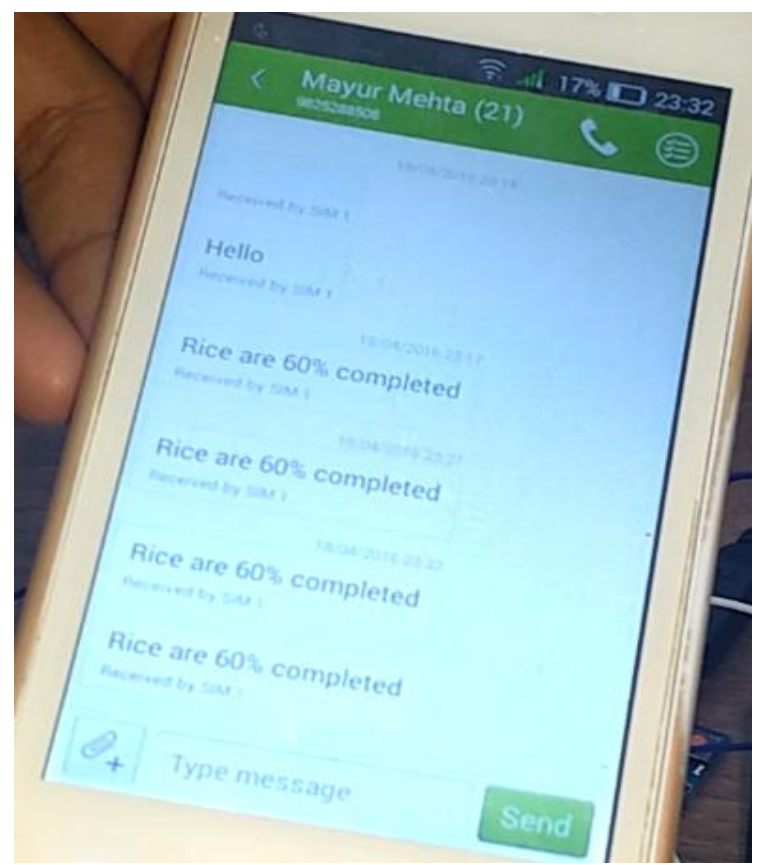

Fig 6: Output on Mobile Phone

\section{CONCLUSION}

With the proposed methodology, the results of experiment encouraged to impart the real time working application. Product management in shopping malls can improve their management without hiring more employees. Also, this methodology can be adopted in houses for the products which are kept in large containers. In future, this methodology is proposed to be modified using Wi-Fi module to communicate in LAN or over the internet. Use of this smart system can be done by various shopping malls or mega malls and store who keep their things in large containers. Even it can be used at home. After implementation, this system can automatically track the product quantity and inform the respect authority whenever it is needed which will reduce the hectic work.

\section{FUTURE ENHANCEMENTS}

The system can be modified by replacing GSM modem with Wi-Fi module. Instead of GSM modem, the alert message can be sent via LAN or the internet by using Wi-Fi module [5]. This Wi-Fi module is comparatively cost effective as its cost less than GSM modem and charges are not applicable on sending every SMS.
Additional functionality, one can make the system even smarter and place an order for the respective product over Ecommerce website. As data is transferred via LAN or the internet to a user, it can also search the product over Ecommerce websites and compare prices and place the order accordingly. This can be possible using Search Engine Optimization (SEO) concepts.

\section{REFERENCES}

[1] S.S.Navghane, M.S.Killedar, Dr.V.M.Rohokale, "IoT Based Smart Garbage and Waste Collection Bin", International Journal of Advanced Research in Electronics and Communication Engineering (IJARECE), 2016, pp. 1576-1578.

[2] Monika K A, Nikitha Rao, Prapulla S B, Shobha G, "Smart Dustbin-An Efficient Garbage Monitoring System", International Journal of Engineering Science and Computing (IJESC), 2016, pp. 7113-7116.

[3] Vikrant Bhor, Pankaj Morajkar, Maheshwar Gurav, Dishant Pandya, "Smart Garbage Management System"International Journal of Engineering Research \& Technology (IJERT), 2015, pp. 1117-1120.

[4] Kiran Patil, Dr. S. L. Lahudkar, "Analysis of MAC Layer Issue using Application Layer Protocol for Smart Waste Management System", IJSTE-International Journal of Science Technology \& Engineering, 2016, pp. 223-231.

[5] Jetendra Joshi,Akshay,Amrit Bagga,Rahul Aggarwal,Manash Deka,Urjit, Abhinav, "SmartGarbage Monitoring System", 7th International Conference on Computing Communication and Networking Technologies, 2016.

[6] Microtronics Technologies, "GSM based garbage and waste collection bins overflow indicator", September 2013.

[7] Basavaraju S R, "Automatic Smart Parking System using Internet of Things (IOT)", International Journal of Scientific and Research Publications, 2015, pp. 629-632.

[8] Vishwajeet H. Bhide, "A Survey on the Smart Homes using Internet of Things (IoT)", International Journal of Advance Research in Computer Science and Management Studies, 2014, pp. 243-246.

[9] Dr. Ovidiu Vermesan and Dr. Peter Friess, 2014, Internet of Things- From Research and Innovation to Market Development. 